\title{
Complex regional pain syndrome type 2
}

INSERM

\section{Source}

INSERM. (1999). Orphanet: an online rare disease and orphan drug data base. Complex regional pain syndrome type 2. ORPHA:99994

Complex regional pain syndrome type 2 (CRPS2), or causalgia is a form of complex regional pain syndrome that develops after damage to a peripheral nerve and is characterized by spontaneous pain, allodynia and hyperalgesia, not necessarily limited to the territory of the injured nerve, as well as at some point, edema, changes in skin blood flow or sudomotor dysfunction in the pain area. 